\title{
A Semianalytical Method for Nonlinear Vibration of Euler-Bernoulli Beams with General Boundary Conditions
}

\author{
Jian-She Peng, ${ }^{1}$ Yan Liu, ${ }^{2}$ and Jie Yang ${ }^{3}$ \\ ${ }^{1}$ School of Industrial Manufacturing, Chengdu University, Sichuan 610106, China \\ ${ }^{2}$ Department of Physics and Electronic Information, China West Normal University, \\ Nanchong, Sichuan 637002, China \\ ${ }^{3}$ School of Aerospace, Mechanical and Manufacturing Engineering, RMIT University, \\ P.O. Box 71, Bundoora, Victoria 3083, Australia
}

Correspondence should be addressed to Jie Yang, j.yang@rmit.edu.au

Received 31 December 2009; Revised 17 April 2010; Accepted 3 June 2010

Academic Editor: Carlo Cattani

Copyright (c) 2010 Jian-She Peng et al. This is an open access article distributed under the Creative Commons Attribution License, which permits unrestricted use, distribution, and reproduction in any medium, provided the original work is properly cited.

This paper presents a new semianalytical approach for geometrically nonlinear vibration analysis of Euler-Bernoulli beams with different boundary conditions. The method makes use of Linstedt-Poincaré perturbation technique to transform the nonlinear governing equations into a linear differential equation system, whose solutions are then sought through the use of differential quadrature approximation in space domain and an analytical series expansion in time domain. Validation of the present method is conducted in numerical examples through direct comparisons with existing solutions, showing that the proposed semianalytical method has excellent convergence and can give very accurate results at a long time interval.

\section{Introduction}

Geometrically nonlinear vibration of beams with different boundary conditions has long been a subject receiving numerous research efforts, as evidenced by many analytical and numerical studies reported in the open literature. Woinowsky-Krieger [1] used the elliptic integral function to solve the nonlinear equation of motion for simply supported beams with immovable ends. Lewandowski [2] applied the Rayleigh-Ritz technique to the nonlinear vibration of beams. A comprehensive review in this field was given by Sathyamoorthy [3]. Sze et al. [4] applied incremental harmonic balance method for nonlinear vibration of axially moving beams. Gadagi and Benaroya [5] studied the dynamic response of an axially loaded tendon of a tension leg platform. Ibrahim and Somnay [6] solved the nonlinear dynamic analysis of an elastic beam isolator sliding on frictional supports. Ozkaya and Tekin 
[7] analyzed the nonlinear vibrations of stepped beam system under different boundary conditions. Chen et al. [8] put forward the multidimensional Lindstedt-Poincare method for nonlinear vibration of axially moving beams. It is noted that many numerical investigations published so far employed step-by-step iterative time integration schemes to calculate the dynamic deflection response which requires a very small time step size in order to achieve the response with sufficient accuracy. This is inevitably computationally expensive. Another big concern associated with these iterative schemes is the error accumulation in each time step which may cause a huge loss of accuracy and sometimes numerical stability problem of the results in later time steps. To overcome this problem, several analytical and semianalytical approaches to the nonlinear vibration of beams which do not involve step-by-step time integration have been proposed; see, for example, those by Azrar et al. [9, 10], Leung and his coworkers [11-13], Lewandowski [14, 15], Nayfeh and his associates [16-18], and Ribeiro [19], to name just a few.

The differential quadrature method (DQM) is an efficient numerical approach developed by Bellman et al. [20] to solve linear and nonlinear differential equations. This method was later introduced to the structural analysis by Bert and his coworkers [21] and has been widely used in many structural engineering problems, including those in the nonlinear vibration of beams, plates, and shells; see, for example, the work by Zhong and Guo [22], Yang et al. [23, 24], Manaoach and Ribeiro [25], Hsu [26], and Tomasiello [27], among many others. Based on DQM approximation, this paper proposes a new semianalytic method for the geometrically nonlinear vibration of Euler-Bernoulli beams with different boundary conditions. The proposed method overcomes the disadvantage of error accumulation that occurs in conventional numerical methods involving iterative time integration and is therefore accurate and numerically stable even for a long time interval. The present paper is structured as follows: Section 2 briefly outlines the DQM rules. Section 3 gives a detailed description of mathematic formulations of the proposed semianalytical approach, starting with the transformation of the nonlinear governing equations into a group of linear differential equations by Linstedt-Poincare perturbation procedure which is followed by the semianalytical solution process for each perturbation equation by using DQM in space domain and an analytical series in the time domain. Section 4 presents some numerical results to validate the proposed method in both accuracy and convergence aspects through direct comparisons between the present results and existing solutions. Some concluding remarks are summarized in Section 4.

\section{Mathematical Formulations}

\subsection{Perturbation Equations}

Consider an isotropic, homogeneous slender beam of length $L$ with uniform cross-section area subjected to a dynamic transverse load $\bar{q}(x, \bar{t})$. Let $\bar{u}$ and $\bar{w}$ be the displacements parallel to the $x$ - and $y$-axes, $\bar{t}$ the time, and $m$ denote the mass density. Based on classical Euler-Bernoulli beam theory and von-Karman nonlinear displacement-strain relationship, the geometrically nonlinear governing equations for flexural vibration of the beam can be derived as

$$
\begin{gathered}
\frac{\partial}{\partial x}\left[E \bar{A}\left(\frac{\partial \bar{u}}{\partial x}+\frac{1}{2}\left(\frac{\partial \bar{w}}{\partial x}\right)^{2}\right)\right]=0, \\
E I \frac{\partial^{4} \bar{w}}{\partial x^{4}}-E \bar{A}\left[\frac{\partial \bar{u}}{\partial x}+\frac{1}{2}\left(\frac{\partial \bar{w}}{\partial x}\right)^{2}\right] \frac{\partial^{2} \bar{w}}{\partial x^{2}}+m \frac{\partial^{2} \bar{w}}{\partial \bar{t}^{2}}=\bar{q}(x, \bar{t}),
\end{gathered}
$$


where $E$ is the elastic modulus of the beam, and $\bar{A}$ and $I$ are the area and the second moment of the cross section. The present analysis considers general boundary conditions, that is, the beam end may be either simply supported or clamped, with the following boundary conditions:

$$
\begin{gathered}
\bar{u}=0, \quad \bar{w}=0, \quad \bar{M}_{x}=0 \quad \text { (simply supported), } \\
\bar{u}=0, \quad \bar{w}=0, \quad \frac{d \bar{w}}{d x}=0 \quad \text { (clamped) }
\end{gathered}
$$

where $\bar{M}_{x}$ is the bending moment of the beam. To facilitate the solution process of (2.1) by using the differential quadrature method in space domain, the following quantities are introduced:

$$
\begin{gathered}
u=\frac{\bar{u}}{\eta}, \quad w=\frac{\bar{w}}{\rho}, \quad \xi=\frac{x}{L}, \quad t=\omega \bar{t}, \quad q=\frac{L^{4} \bar{q}}{\rho E I}, \quad q=\varepsilon F, \\
p=\frac{\omega}{\omega_{L}}, \quad \omega_{L}=\left(\frac{1}{L}\right)^{2} \sqrt{\frac{E I}{m}}, \quad \rho^{2}=\frac{I}{\bar{A}}
\end{gathered}
$$

where $\omega_{L}$ and $\omega$ refer to the linear and nonlinear frequencies of the beam, respectively, and $\varepsilon$ is the small perturbation parameter. Substituting these quantities into (2.1) leads to dimensionless nonlinear governing equations as below

$$
\begin{gathered}
\frac{\partial^{2} u}{\partial \xi^{2}}+\frac{\partial w}{\partial \xi} \frac{\partial^{2} w}{\partial \xi^{2}}=0 \\
\frac{\partial^{4} w}{\partial \xi^{4}}-\left[\frac{\partial u}{\partial \xi}+\frac{1}{2}\left(\frac{\partial w}{\partial \xi}\right)^{2}\right] \frac{\partial^{2} w}{\partial \xi^{2}}+p^{2} \frac{\partial^{2} w}{\partial t^{2}}=\varepsilon F .
\end{gathered}
$$

Deflection $w$, axial displacement $u$, and frequency parameter $p$ can be expanded into series forms in terms of a small perturbation parameter $\varepsilon$

$$
\begin{gathered}
w=w_{1} \varepsilon+w_{3} \varepsilon^{3}+w_{5} \varepsilon^{5}+\cdots, \\
u=u_{2} \varepsilon^{2}+u_{4} \varepsilon^{4}+\cdots \\
p^{2}=1+\varepsilon^{2} \sigma_{2}+\varepsilon^{4} \sigma_{4}+\cdots
\end{gathered}
$$

Inserting (2.5)-(2.7) into (2.4), and equating the terms of the same power of $\varepsilon$ yield a series 
of perturbation equations

$$
\begin{aligned}
& \varepsilon^{1}: \quad \frac{\partial^{4} w_{1}}{\partial \xi^{4}}+\frac{\partial^{2} w_{1}}{\partial t^{2}}=F \\
& \varepsilon^{2}: \quad \frac{\partial^{2} u_{2}}{\partial \xi^{2}}+\frac{\partial w_{1}}{\partial \xi} \frac{\partial^{2} w_{1}}{\partial \xi^{2}}=0 \\
& \varepsilon^{3}: \quad \frac{\partial^{4} w_{3}}{\partial \xi^{4}}-\frac{\partial u_{2}}{\partial \xi} \frac{\partial^{2} w_{1}}{\partial \xi^{2}}-\frac{1}{2}\left(\frac{\partial w_{1}}{\partial \xi}\right)^{2} \frac{\partial^{2} w_{1}}{\partial \xi^{2}}+\frac{\partial^{2} w_{3}}{\partial t^{2}}+\sigma_{2} \frac{\partial^{2} w_{1}}{\partial t^{2}}=0 \\
& \varepsilon^{4}: \quad \frac{\partial^{2} u_{4}}{\partial \xi^{2}}+\frac{\partial w_{1}}{\partial \xi} \frac{\partial^{2} w_{3}}{\partial \xi^{2}}+\frac{\partial w_{3}}{\partial \xi} \frac{\partial^{2} w_{1}}{\partial \xi^{2}}=0
\end{aligned}
$$

The initial conditions considered in the present study are

$$
\begin{gathered}
w_{1}(\xi, 0)=w_{10}, \quad \frac{\partial w_{1}(\xi, 0)}{\partial t}=0 \\
w_{3}(\xi, 0)=0, \quad \frac{\partial w_{3}(\xi, 0)}{\partial t}=0
\end{gathered}
$$

where $w_{10}$ refers to initial vibration amplitude. By making use of the dimensionless quantities and perturbation series defined above, the dimensionless form of the associated boundary conditions in each perturbation can also be obtained.

\subsection{Differential Quadrature Method}

According to the DQM rule, an unknown function $g(x)$ and its $n$th order derivative with respect to $x$ can be approximated as the weighted linear sums of its function values at a number of sampling points in the $x$-axis as

$$
\begin{gathered}
g(x)=\sum_{i=1}^{N} g\left(x_{i}\right) L_{i}(x), \\
\left.\frac{d^{n} g(x)}{d x^{n}}\right|_{x=x_{i}}=\sum_{j=1}^{N} C_{i j}^{(n)} g\left(x_{j}\right) \quad(n=1, \ldots, N-1),
\end{gathered}
$$

where $N$ is the total number of sampling points, $L_{i}(x)$ are Lagrange interpolation polynomials, and the weighting coefficients $C_{i j}^{(n)}$ can be calculated from recursive formulae $[20,21]$. 


\subsection{Semianalytical Solution of the 1st-Order Perturbation Equation}

This study is focused on the nonlinear vibration due to an initial deflection. In such a case, the right-hand-side term in (2.8) $F=0$. Hence, the first-order perturbation becomes

$$
\frac{\partial^{4} w_{1}}{\partial \xi^{4}}+\frac{\partial^{2} w_{1}}{\partial t^{2}}=0
$$

The solution of (2.16) can be readily obtained by separation of variables

$$
w_{1}(\xi, t)=\sum_{m=0}^{N_{s}} T_{1 m}(t) \phi_{1 m}(\xi)
$$

where $N_{s}$ is the number of terms in the truncated time series,

$$
T_{1 m}(t)=\cos \left(p_{m} t\right)
$$

is the solution in the time domain for the first-order perturbation equation, and $p_{m}$ and $\delta_{1 m}(\xi)$ are the frequency parameter and the associated mode shape function.

The transient deflection at an arbitrary sampling point " $i$ " can be expressed as a cosine series with a supplementary term as

$$
w_{1}\left(\xi_{i}, t\right)=\delta_{0}^{i}+\sum_{m=1}^{N_{s}} T_{1 m}(t) \delta_{1 m}^{i} \quad(i=1, \ldots, N)
$$

where $\delta_{1 m}^{i}=\phi_{1 m}\left(\xi_{i}\right)$ needs to be determined. The frequency parameter $p_{m}$ of beams with different boundary conditions can be written in a general form as

$$
p_{m}=[(m+j) \pi]^{2}
$$

in which $j=0$ for a simply supported beam, $j=1 / 2$ for a clamped beam, $j=-1 / 2$ for a beam clamped at one end and free at the other end, and $j=1 / 4$ for a beam clamped at one end but simply supported at the other end.

Since $w_{1}\left(\xi_{i}, t\right)$ needs to satisfy the initial conditions in (2.12), one has

$$
\delta_{0}^{i}=w_{10}^{i}-\sum_{m=1}^{N} \delta_{1 m}^{i}
$$

The time domain function in (2.19) can be readily obtained as

$$
w_{1}\left(\xi_{i}, t\right)=w_{10}^{i}+\sum_{m=1}^{N_{s}}\left(\cos p_{m 1} t-1\right) \quad \delta_{1 m}^{i} \quad(i=1, \ldots, N)
$$


Differentiation of (2.22) with respect to time twice gives

$$
\ddot{w}_{1}\left(\xi_{i}, t\right)=-\sum_{m=1}^{N_{s}} p_{m}^{2}\left(\cos p_{m 1} t\right) \delta_{1 m}^{i} \quad(i=1, \ldots, N)
$$

The deflection response of the 1st-order perturbation equation can be determined by

$$
w_{1}(\xi, t)=\sum_{i=1}^{N} w_{1}\left(\xi_{i}, t\right) L_{i}(\xi)
$$

Substitution of (2.23) and application of DQM approximation (2.15) to (2.16) result in a semianalytical algebraic equation at an arbitrary sampling point

$$
\begin{array}{r}
\sum_{j=1}^{N} C_{i j}^{(4)} \sum_{m=1}^{N_{s}}\left(\cos p_{m} t_{k}-1\right) \delta_{1 m}^{j}-p_{1}^{2} \sum_{m=1}^{N_{s}} p_{m}^{2} \cos \left(p_{m} t_{k}\right) \delta_{1 m}^{i}=-\sum_{j=1}^{N} C_{i j}^{(4)} w_{10}^{j} \\
\left(i=1, \ldots, N ; k=1, \ldots, N_{s}\right) .
\end{array}
$$

This linear equation system can be written in a matrix form as

$$
\left[D_{1}\right]\left\{\delta_{1}\right\}=\left\{f_{1}\right\}
$$

where $\left[D_{1}\right]$ and $\left\{\delta_{1}\right\}$ are the coefficient matrix and unknown vector to be solved, and $\left\{f_{1}\right\}$ is the generalized load vector due to initial deflection. Once $\left\{\delta_{1}\right\}$ is determined from (2.26), the deflections at all of the sampling points can be calculated from (2.22) and the 1st-order deflection solution of the beam is then obtained from (2.24).

\subsection{Semianalytical Solution of the 2nd-Order Perturbation Equation}

To solve the 2nd-order perturbation equation (2.9), the unknown axial displacement $u_{2}$ is expressed as

$$
u_{2}(\xi, t)=\sum_{m=1}^{N_{s}} T_{2 m}(t) \phi_{2 m}(\xi)
$$

Substituting the 1st-order deflection solution and (2.27) into (2.9) yields

$$
\sum_{m=1}^{N_{S}} T_{2 m}(t) \frac{d^{2} \phi_{2 m}}{d \xi^{2}}=-\sum_{m=1}^{N_{S}} \sum_{n=1}^{N_{S}} T_{1 m}(t) T_{1 n}(t) \frac{d \phi_{1 m}}{d \xi} \frac{d^{2} \phi_{1 n}}{d \xi^{2}}
$$


This gives

$$
T_{2 m}=-\frac{\sum_{n=1}^{N_{s}} T_{1 m} T_{1 n}\left(d \phi_{1 m} / d \xi\right)\left(d^{2} \phi_{1 n} / d \xi^{2}\right)}{d^{2} \phi_{2 m} / d \xi^{2}} .
$$

Therefore, (2.27) becomes

$$
u_{2}(\xi, t)=\sum_{m=1}^{N s} \sum_{n=1}^{N s} T_{1 m} T_{1 n} \psi_{2 m n}(\xi)
$$

with

$$
\psi_{2 m n}(\xi)=-\frac{\left(d \phi_{1 m} / d \xi\right)\left(d^{2} \phi_{1 n} / d \xi^{2}\right)}{d^{2} \phi_{2 m} / d \xi^{2}} \phi_{2 m}
$$

From (2.30), the axial displacement $u_{2}$ at an arbitrary sampling point " $i$ " is

$$
u_{2}\left(\xi_{i}, t\right)=\sum_{m=1}^{N_{s}} \sum_{n=1}^{N_{s}} T_{1 m}(t) T_{1 n}(t) \delta_{2 m n}^{i}
$$

Putting (2.30) into (2.9) and then applying DQM approximation, one has

$$
\sum_{j=1}^{N} C_{i j}^{(2)} \sum_{m=1}^{N_{s}} \sum_{n=1}^{N_{s}} T_{1 m} T_{1 n} \delta_{2 m n}^{j}=-\sum_{j=1}^{N} C_{i j}^{(1)} \sum_{m=1}^{N_{s}} T_{1 m} \delta_{1 m}^{j} \sum_{k=1}^{N} C_{i k}^{(2)} \sum_{n=1}^{N_{s}} T_{1 n} \delta_{1 n}^{k} \quad(i=1, \ldots, N)
$$

in a matrix form

$$
\left[D_{2}\right] \cdot\left\{\delta_{2}\right\}=\left\{f_{2}\right\}
$$

Note that the right-hand-side term $\left\{f_{2}\right\}$ is the pseudodynamic force vector totally dependent on the 1st-order deflection solution already obtained. After the unknown vector $\left\{\delta_{2}\right\}$ composed of $\delta_{2 m n}^{j}$ is determined from (2.34), $u_{2}$ can be calculated from the relationship

$$
\psi_{2 m n}(\xi)=\sum_{i=1}^{N} \delta_{2 m n}^{i} L_{i}(\xi)
$$

and (2.30). 


\subsection{Semianalytical Solution of the 3rd-Order Perturbation Equation}

The 3rd-order perturbation equation (2.10) can be rewritten as

$$
\frac{\partial^{4} w_{3}}{\partial \xi^{4}}+\frac{\partial^{2} w_{3}}{\partial t^{2}}=f(\xi, t)
$$

where the right-hand-side term

$$
f(\xi, t)=\left\{\left[\frac{\partial u_{2}}{\partial \xi}+\frac{1}{2}\left(\frac{\partial w_{1}}{\partial \xi}\right)^{2}\right]\right\} \frac{\partial^{2} w_{1}}{\partial \xi^{2}}-\sigma_{2} \frac{\partial^{2} w_{1}}{\partial t^{2}}
$$

Substituting (2.17) and (2.30) into (2.37) gives

$$
f(\xi, t)=\sum_{l=1}^{N_{s}} \sum_{m=1}^{N_{S}} \sum_{n=1}^{N_{s}} T_{1 l} T_{1 m} T_{1 n} \varphi_{l m n}+\sum_{n=1}^{N_{s}} p_{n}^{2} \sigma_{2} T_{1 n} \phi_{1 n},
$$

where

$$
\varphi_{l m n}(\xi)=\left\{\frac{d \psi_{2 l m}}{d \xi}+\frac{1}{2}\left[\frac{d \phi_{1 l}}{d \xi} \frac{d \phi_{1 m}}{d \xi}\right]\right\} \frac{d^{2} \phi_{1 n}}{d \xi^{2}}
$$

The solution of (2.36) can be readily obtained by the method of separation of variables

$$
w_{3}(\xi, t)=\sum_{r=1}^{N_{s}} T_{3 r}(t) \phi_{3 r}(\xi)
$$

It is evident from (2.16) and (2.36) that $\phi_{3 m}(\xi)$ is the same as $\phi_{1 m}(\xi)$. Therefore,

$$
w_{3}(\xi, t)=\sum_{r=1}^{N_{s}} T_{3 r}(t) \phi_{1 r}(\xi)
$$

and (2.36) becomes

$$
\sum_{r=1}^{N_{s}} T_{3 r}(t) \frac{\partial^{4} \phi_{1 r}}{\partial \xi^{4}}+\sum_{r=1}^{N_{s}} \frac{\partial^{2} T_{3 r}}{\partial t^{2}} \phi_{1 r}=f(\xi, r)
$$

From (2.16)-(2.18), one has

$$
\frac{\partial^{4} \phi_{1 r}}{\partial \xi^{4}}=P_{r}^{2} \phi_{1 r}
$$


Expanding $f(\xi, t)$ into Fourier series in function $\phi_{1 m},(2.42)$ becomes

$$
\sum_{r=1}^{N_{s}}\left[T_{3 r}^{\prime \prime}+T_{3 r} p_{r}^{2}\right] \phi_{1 r}(\xi)=\sum_{r=1}^{N_{s}} f_{r}(\xi, t) \phi_{1 r}(\xi)
$$

which yields

$$
T_{3 r}^{\prime \prime}+T_{3 r} p_{r}^{2}=f_{r}(\xi, t)
$$

where right-hand-side term

$$
\begin{gathered}
f_{r}(t)=\frac{1}{N_{3 r}^{2}} \int_{0}^{1} f(\xi, t) \phi_{1 r}(\xi) d \xi=\sum_{l=1}^{N_{s}} \sum_{m=1}^{N_{s}} \sum_{n=1}^{N_{s}} a_{l m n r} T_{1 l} T_{1 m} T_{1 n}+\sum_{n=1}^{N_{s}} \sigma_{2} b_{n r} p_{n}^{2} T_{1 n} \\
N_{3 r}^{2}=\int_{0}^{1} \phi_{1 r}^{2}(\xi) d \xi, \quad a_{l m n r}=\frac{1}{N_{3 r}^{2}} \int_{0}^{1} \varphi_{l m n}(\xi) \phi_{1 r}(\xi) d \xi, \quad b_{n r}=\frac{1}{N_{3 r}^{2}} \int_{0}^{1} \phi_{1 n}(\xi) \phi_{1 r}(\xi) d \xi .
\end{gathered}
$$

Since $\phi_{1 r}$ are orthogonal functions, we have

$$
b_{n r}= \begin{cases}0, & n \neq r, \\ \frac{1}{N_{3 r}^{2}} \int_{0}^{1} \phi_{1 r} \cdot \phi_{1 r}(\xi) d \xi=1, & n=r .\end{cases}
$$

To remove the secular terms in $(2.43), f_{3 m}(t)$ is expanded as

$$
\begin{aligned}
& f_{r}(t)=\left\{\sum_{l=1}^{N s} \sum_{m=1}^{N s} \sum_{n=1}^{N s} \cos \left(p_{l}+p_{m}+p_{n}\right) t a_{l m n r}+\sum_{l=1}^{N s} \sum_{m=1}^{N s} \sum_{n=1}^{N s} l \neq n, n \neq m \cos \left(p_{l}+p_{m}-p_{n}\right) t a_{l m n r}\right. \\
& +\sum_{l=1}^{N s} \sum_{m=1}^{N s} \sum_{n=1}^{N s} l \neq m, m \neq n \cos \left(p_{l}-p_{m}+p_{n}\right) t a_{l m n r}
\end{aligned}
$$

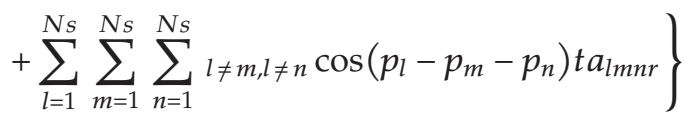

$$
\begin{aligned}
& +\left\{2 \sum_{l=1}^{N s} \sum_{m=1}^{N s}{ }_{l=n} \cos p_{m} t a_{l m l r}+2 \sum_{l=1}^{N s} \sum_{m=1}^{N s}{ }_{m=n} \cos p_{l} t a_{l m m r}+2 \sum_{l=1}^{N s} \sum_{n=1}^{N s}{ }_{l=m} \cos p_{n} t a_{l l n r}\right. \\
& \left.+3 \sum_{l=1}^{N s}{ }_{l=m=n} \cos p_{l} t a_{l l l r}\right\}+p_{r}^{2} \sigma_{2 r} \cos p_{r} t .
\end{aligned}
$$


The following condition must be satisfied in order to remove the last two terms in (2.48),

$$
\begin{aligned}
& \left\{2 \sum_{l=1}^{N s} \sum_{m=1}^{N s}{ }_{l=n} \cos p_{m} t a_{l m l r}+2 \sum_{l=1}^{N s} \sum_{m=1}^{N s}{ }_{m=n} \cos p_{l} t a_{l m m r}+2 \sum_{l=1}^{N s} \sum_{n=1}^{N s}{ }_{l=m} \cos p_{n} t a_{l l n r}\right. \\
& \left.+3 \sum_{l=1}^{N s}{ }_{l=m=n} \cos p_{l} t a_{l l l r}\right\}+p_{r}^{2} \sigma_{2 r} \cos p_{r} t=0
\end{aligned}
$$

The sum of the coefficients for $\cos \left(p_{r} t\right)$ must be zero, which gives the expressions of $\sigma_{2}$ for different values of $m$ as

$$
\sigma_{2 r}=\frac{-1}{p_{r}^{2}}\left\{2 \sum_{m=1}^{N s}\left[a_{m r m r}+a_{r m m r}+a_{m m r r}\right]+3 a_{r r r}\right\}
$$

Thus the term $f_{3 m}(t)$ without secular terms is

$$
\begin{aligned}
f_{r}(t)=\{ & \sum_{l=1}^{N s} \sum_{m=1}^{N s} \sum_{n=1}^{N s} \cos \left(p_{l}+p_{m}+p_{n}\right) t a_{l m n r}+\sum_{l=1}^{N s} \sum_{m=1}^{N s} \sum_{n=1}^{N s} l \neq n, n \neq m \cos \left(p_{l}+p_{m}-p_{n}\right) t a_{l m n r} \\
& +\sum_{l=1}^{N S} \sum_{m=1}^{N s} \sum_{n=1}^{N s} l \neq m, m \neq n \cos \left(p_{l}-p_{m}+p_{n}\right) t a_{l m n r} \\
& \left.+\sum_{l=1}^{N S} \sum_{m=1}^{N S} \sum_{n=1}^{N s} l \neq m, l \neq n \cos \left(p_{l}-p_{m}-p_{n}\right) t \cdot a_{l m n r}\right\} .
\end{aligned}
$$

Equation (2.45) can then be rewritten as

$$
T_{3 r}^{\prime \prime}+T_{3 r} p_{r}^{2}=f_{r}(t)
$$

From (2.13), the initial condition for (2.52) can be derived by using separation of variable method

$$
T_{3 r}(0)=\frac{d T_{3 r}(0)}{d t}=0
$$


The solution of (2.52) under the given initial condition (2.53) can be obtained through Laplace transform as

$$
\begin{aligned}
& T_{3 r}(t)= \sum_{l=1}^{M} \sum_{m=1}^{M} \sum_{n=1}^{M} \frac{\cos \left(p_{r} t\right)-\cos \left(\left(p_{l}+p_{m}+p_{n}\right) t\right)}{\left(p_{l}+p_{m}+p_{n}\right)^{2}-p_{r}^{2}} \gamma_{l m n r} \\
&+\sum_{l=1}^{M} \sum_{m=1}^{M} \sum_{n=1}^{M} l_{l \neq n, n \neq m} \frac{\cos \left(p_{r} t\right)-\cos \left(\left(p_{l}+p_{m}-p_{n}\right) t\right)}{\left(p_{l}+p_{m}-p_{n}\right)^{2}-p_{r}^{2}} \gamma_{l m n r} \\
&+\sum_{l=1}^{M} \sum_{m=1}^{M} \sum_{n=1}^{M} l_{l \neq m, m \neq n} \frac{\cos \left(p_{r} t\right)-\cos \left(\left(p_{l}-p_{m}+p_{n}\right) t\right)}{\left(p_{l}-p_{m}+p_{n}\right)^{2}-p_{r}^{2}} \gamma_{l m n r} \\
&+\sum_{l=1}^{M} \sum_{m=1}^{M} \sum_{n=1}^{M}{ }_{l \neq m, l \neq n} \frac{\cos \left(p_{r} t\right)-\cos \left(\left(p_{l}-p_{m}-p_{n}\right) t\right)}{\left(p_{l}-p_{m}-p_{n}\right)^{2}-p_{r}^{2}} \gamma_{l m n r} \\
&\left(\text { where } \gamma_{l m n r}=\frac{1}{4} a_{l m n r}\right) .
\end{aligned}
$$

Finally, we have

$$
w_{3}(\xi, t)=\sum_{r=1}^{N s} T_{3 r}(t) \phi_{1 r}(\xi)
$$

The deflections response of nonlinear free vibration of the beam is then calculated from

$$
w=w_{1} \varepsilon+w_{3} \varepsilon^{3}+\cdots
$$

while the nonlinear frequency response, with higher-order terms being neglected, is given by (2.50) and (2.7) as

$$
p_{r}^{2}=1+\varepsilon^{2} \sigma_{2 r}
$$

and the nonlinear frequency response can be obtained from

$$
\left(\frac{\omega_{r}}{\omega_{A}}\right)^{2}=1-\varepsilon^{2} \frac{1}{p_{r}^{2}}\left\{2 \sum_{m=1}^{N s}\left[a_{m r m r}+a_{r m m r}+a_{m m r r}\right]+3 a_{r r r r}\right\} .
$$

\section{Numerical Results}

To illustrate its efficiency and accuracy, the proposed semianalytical approach is used to study the nonlinear frequency and dynamic response of Euler-Bernoulli beams of rectangular cross section $(b \times h=0.01 \mathrm{~m} \times 0.02 \mathrm{~m})$ under various boundary conditions with a given 
Table 1: Normalized frequency ratio $\omega_{n} / \omega_{L}$ for a simply supported beam.

\begin{tabular}{cccccc}
\hline$A$ & $N=9$ & $N=11$ & $N=13$ & Woinowsky-Krieger [1] & Mei [28] \\
\hline 0.1 & 1.0009 & 1.0009 & 1.0009 & 1.0008 & 1.0009 \\
0.2 & 1.0037 & 1.0037 & 1.0037 & 1.0038 & 1.0037 \\
0.4 & 1.0149 & 1.0149 & 1.0149 & 1.0150 & 1.0148 \\
0.6 & 1.0331 & 1.0331 & 1.0334 & 1.0380 & 1.0339 \\
0.8 & 1.0580 & 1.0581 & 1.0588 & 1.0580 & 1.0578 \\
1.0 & 1.0891 & 1.0893 & 1.0908 & 1.0890 & 1.0889 \\
1.5 & 1.1893 & 1.1910 & 1.1932 & 1.1900 & 1.1902 \\
2.0 & 1.3140 & 1.3201 & 1.3300 & 1.3160 & 1.3022 \\
\hline
\end{tabular}

Table 2: Normalized frequency ratio $\omega_{n} / \omega_{L}$ for a clamped beam.

\begin{tabular}{ccccc}
\hline$A$ & $N=9$ & $N=11$ & $N=13$ & Mei [28] \\
\hline 0.1 & 1.0003 & 1.0003 & 1.0003 & 1.0003 \\
0.2 & 1.0012 & 1.0012 & 1.0012 & 1.0012 \\
0.4 & 1.0050 & 1.0047 & 1.0048 & 1.0048 \\
0.6 & 1.0112 & 1.0106 & 1.0107 & 1.0107 \\
0.8 & 1.0198 & 1.0187 & 1.0190 & 1.0190 \\
1.0 & 1.0307 & 1.0290 & 1.0295 & 1.0296 \\
1.5 & 1.0676 & 1.0640 & 1.0650 & 1.0653 \\
2.0 & 1.1169 & 1.1100 & 1.1125 & 1.1135 \\
\hline
\end{tabular}

initial vibration amplitude and zero-valued initial velocity. The dimensionless vibration amplitude is defined as $A=W / \rho$ (or $A=6 W / \sqrt{3} h$ for a rectangular beam) where $W$ is the vibration amplitude at the midpoint of the beam. The parameters used herein are $L=0.5 \mathrm{~m}, E=150 \mathrm{GPa}$, and $m=8 \times 10^{3} \mathrm{~kg} / \mathrm{m}^{3}$. The results are presented in both tabular and graphical forms in terms of the normalized frequency ratio $\omega_{n} / \omega_{L}$ and dimensionless dynamic deflections at the midpoint of the beam.

Among the sampling point distribution schemes available, a nonuniform distribution is chosen in the present analysis due to its excellent convergence $[21,26]$, that is,

$$
x_{k}=\frac{1}{2}\left(1-\cos \frac{k \pi}{N-1}\right) \quad(k=0,1, \ldots, N-1) .
$$

In what follows, the total number of sampling points in the space domain is $N=11$, unless stated otherwise.

In Figures 1-3, the present solutions and exact solutions are represented by solid lines and circular dots, respectively.

\subsection{Nonlinear Vibration Frequency}

Tables 1, 2, and 3 list, respectively, the normalized fundamental frequency ratios $\omega_{n} / \omega_{L}$ for a simply supported beam, a clamped beam, and a beam simply supported at one end but clamped at the other end at varying dimensionless vibration amplitudes $A=0.1 \sim 2$.0. All beams exhibit typical "hard-spring" behavior, that is, the frequency ratio increases with an increase in vibration amplitude. Comparisons between the results with varying total number 


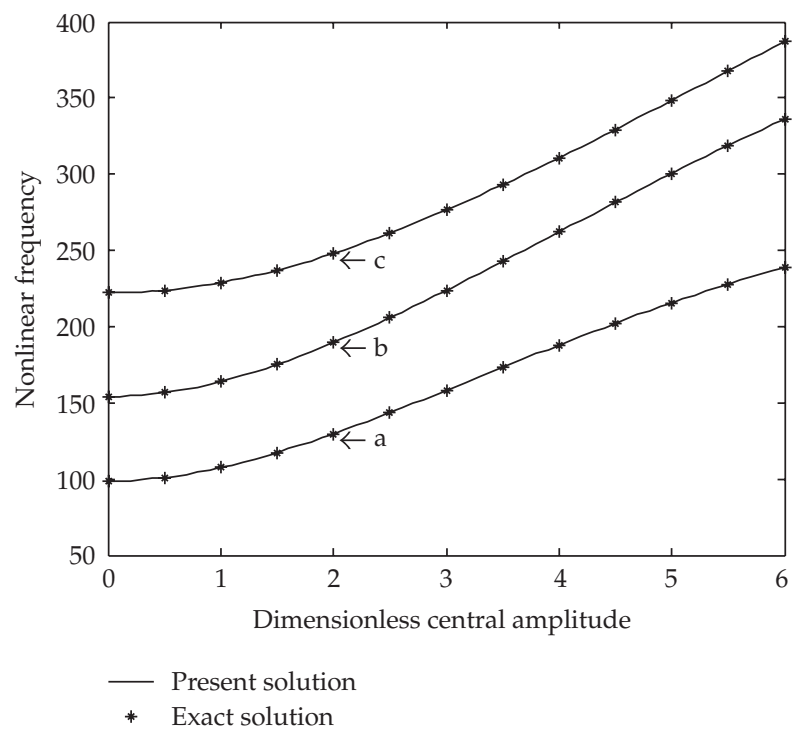

Figure 1: Nonlinear fundamental frequency versus dimensionless central amplitude relationship of beams with different boundary conditions.

of sampling points $N=9,11,13$ show that the present method converges very well to produce very accurate results when $N \geq 11$. Our results are compared with the existing results obtained by using elliptic function [1] and the finite element method [28]. The relative errors, defined as

$$
\frac{(\text { Present Solution }- \text { Existing Solution) }}{\text { Existing Solution }} \times 100 \% \text {, }
$$

are also provided. Excellent agreement can be observed in these tables. The relationship between nonlinear fundamental frequency and dimensionless vibration amplitude $(A=$ $0.0 \sim 6.0$ ) of these three beams are shown in Figure 1 where curves a, b, and c are for a simply supported beam, a beam simply supported at one end but clamped at the other end, and a clamped beam. The scatter points represent the elliptical function results using the formulae provided by Woinowsky-Krieger [1]. As can be seen, the present results and the elliptical function results are almost identical. The clamped beam has the highest fundamental frequency with the lowest deflection while the simply supported beam has the smallest fundamental frequency and the largest deflection. This is because the end support of the clamped beam is much stronger than its simply supported counterpart.

\subsection{Nonlinear Dynamic Response}

Convergence study on the nonlinear dynamic response is conducted in both Tables 4 and 5 where central deflections of a simply supported beam at different time (in sec), calculated by using different total number of sampling points $N$ in the space domain and different number of truncated series terms $N_{s}$ in the time domain, are given and compared with the results calculated by using the formulae given by Woinowsky-Krieger [1]. The dimensionless 


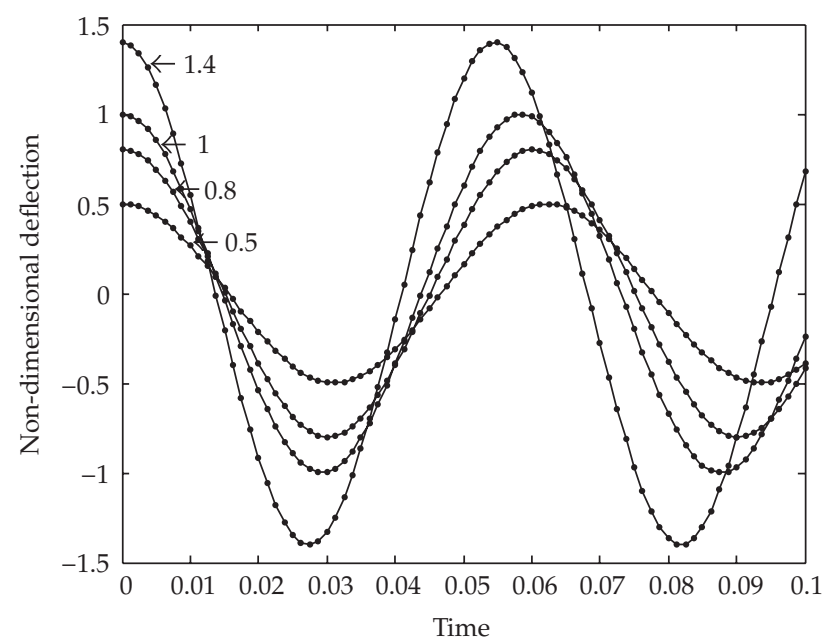

Figure 2: Nondimensional central deflection response of a simply supported beam under different initial vibration amplitudes.

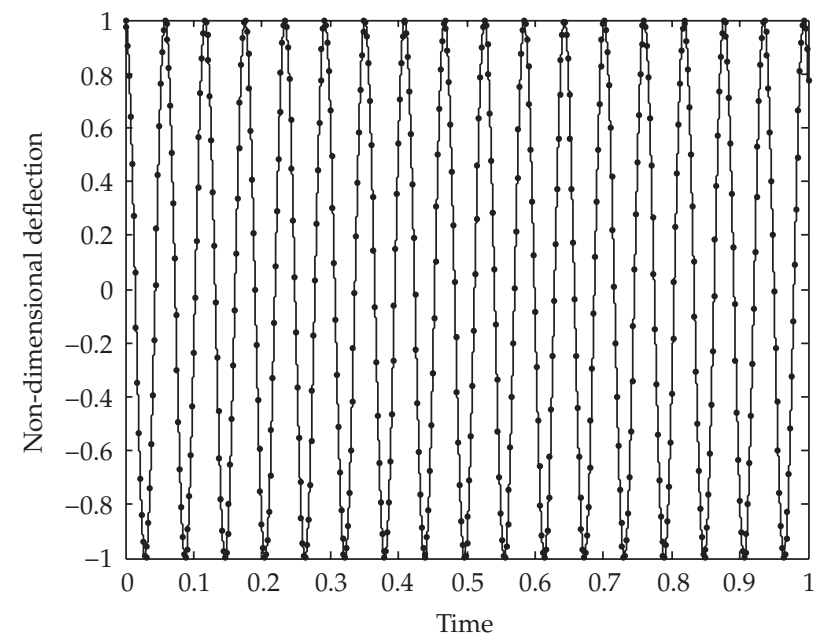

Figure 3: Nondimensional central deflection response of a simply supported beam.

Table 3: Normalized frequency ratio $\omega_{n} / \omega_{L}$ for a beam simply supported at one end and clamped at the other end.

\begin{tabular}{llllc}
\hline$A$ & $N=9$ & $N=11$ & $N=13$ & Mei [28] \\
\hline 0.1 & 1.0006 & 1.0006 & 1.0006 & 1.0006 \\
0.2 & 1.0026 & 1.0026 & 1.0026 & 1.0026 \\
0.4 & 1.0103 & 1.0103 & 1.0103 & 1.0106 \\
0.6 & 1.0230 & 1.0231 & 1.0231 & 1.0238 \\
0.8 & 1.0404 & 1.0406 & 1.0407 & 1.0418 \\
1.0 & 1.0624 & 1.0627 & 1.0628 & 1.0647 \\
1.5 & 1.1346 & 1.1353 & 1.1355 & 1.1404 \\
2.0 & 1.2272 & 1.2283 & 1.2286 & 1.2385 \\
\hline
\end{tabular}


Table 4: Nonlinear central deflection response of a simply supported beam: solutions with varying number of terms in time domain series $N_{s}$.

\begin{tabular}{lccccccc}
\hline Time $(\mathrm{s})$ & $N_{s}=1$ & $N_{s}=3$ & $N_{s}=5$ & Woinowsky-Krieger [1] & Error 1 & Error 2 & Error 3 \\
\hline 0.0050 & 0.8622 & 0.8566 & 0.8553 & 0.8533 & 1.0505 & 0.3895 & 0.2405 \\
0.0100 & 0.4811 & 0.4720 & 0.4700 & 0.4665 & 3.1360 & 1.1828 & 0.7540 \\
0.0150 & -0.0420 & -0.0398 & -0.0395 & -0.0404 & 3.7991 & -1.6178 & -2.2036 \\
0.0300 & -0.9965 & -0.9939 & -0.9942 & -0.9963 & 0.0134 & -0.2437 & -0.2146 \\
0.0400 & -0.4060 & -0.3952 & -0.3938 & -0.3928 & 3.3588 & 0.6230 & 0.2717 \\
0.0450 & 0.1259 & 0.1240 & 0.1231 & 0.1210 & 4.0692 & 2.4780 & 1.7217 \\
0.0550 & 0.9335 & 0.9300 & 0.9297 & 0.9286 & 0.5294 & 0.1543 & 0.1177 \\
0.0650 & 0.7672 & 0.7596 & 0.7574 & 0.7545 & 1.6804 & 0.6677 & 0.3804 \\
0.0750 & -0.2086 & -0.2022 & -0.2010 & -0.2009 & 3.8259 & 0.6691 & 0.0613 \\
0.0850 & -0.9596 & -0.9547 & -0.9549 & -0.9566 & 0.3065 & -0.2001 & -0.1767 \\
0.0900 & -0.9698 & -0.9655 & -0.9657 & -0.9676 & 0.2261 & -0.2173 & -0.1960 \\
0.0950 & -0.7113 & -0.6999 & -0.6983 & -0.6974 & 1.9872 & 0.3496 & 0.1245 \\
0.1000 & -0.2477 & -0.2404 & -0.2390 & -0.2387 & 3.7615 & 0.7006 & 0.1317 \\
\hline
\end{tabular}

vibration amplitude is $A=1.0$. Error 1, Error 2, and Error 3 in Table 4 indicate the relative error percentages between the exact solutions and the present results with $N=9$ and $N_{s}=1$, $N=9$ and $N_{s}=3, N=9$ and $N_{s}=5$ while Error 4, Error 5, and Error 6 in Table 5 are the relative error percentages between the exact solutions and the present results with $N=9$ and $N_{s}=3, N=11$ and $N_{s}=3, N=13$ and $N_{s}=3$. Our semianalytical solutions converge monotonically as $N_{s}$ increases but nonmonotonically with an increasing $N$. However, the present method is capable of giving very accurate results with relative error less than $1.5 \%$ when $N_{s} \geq 3$ and $N \geq 11$. We have calculated the nonlinear dynamic response of beams with other end supports as well and found that the results exhibit the same convergence characteristics as observed in Tables 4 and 5. These results are, therefore, not detailed here.

Figure 2 depicts the nonlinear dynamic central deflection response of a simply supported beam with dimensionless initial deflection $w_{10}=0.5 \sin (\pi \xi), 0.8 \sin (\pi \xi), 1.0 \sin (\pi \xi)$, and $1.4 \sin (\pi \xi)$. The time interval in this example is relatively short, that is, $t=0 \sim 0.1 \mathrm{sec}$. The result within a much longer time interval $t=0 \sim 1 \mathrm{sec}$ is displayed in Figure 3 where $w_{10}=1.0 \sin (\pi \xi) . N=11$ and $N_{s}=3$ are used in these two examples. Again, the present results agree very well with the elliptical function solutions by Woinowsky-Krieger [1]. It is also seen that a bigger initial deflection magnitude results in larger vibration amplitude.

It is worthy of noting that unlike the conventional numerical integration schemes commonly used to calculate dynamic response whose accuracy is quickly degraded at later time steps due to the accumulation of numerical errors, the proposed technique does not involve iterative time integration and accumulative errors and is therefore able to give results with excellent accuracy even for a very long time interval, as demonstrated in Figure 3.

\section{Conclusions}

A new semianalytical method for geometrically nonlinear vibration analysis of EulerBernoulli beams with different boundary conditions is presented in this paper. The proposed method is based on the perturbation technique, differential quadrature approximation, and an analytical series expansion in the time domain. Numerical results show that this 
Table 5: Nonlinear central deflection response of a simply supported beam: solutions with varying total number of sampling points $N$.

\begin{tabular}{lccccccc}
\hline Time $(\mathrm{s})$ & $N=9$ & $N=11$ & $N=13$ & Woinowsky-Krieger [1] & Error 4 & Error 5 & Error 6 \\
\hline 0.0050 & 0.8566 & 0.8562 & 0.8562 & 0.8533 & 0.3895 & 0.3458 & 0.3484 \\
0.0100 & 0.4720 & 0.4711 & 0.4712 & 0.4665 & 1.1828 & 0.9922 & 1.0026 \\
0.0150 & -0.0398 & -0.0410 & -0.0410 & -0.0404 & -1.6178 & 1.4624 & 1.4228 \\
0.0300 & -0.9939 & -0.9967 & -0.9967 & -0.9963 & -0.2437 & 0.0411 & 0.0378 \\
0.0400 & -0.3952 & -0.3969 & -0.3970 & -0.3928 & 0.6230 & 1.0606 & 1.0657 \\
0.0450 & 0.1240 & 0.1223 & 0.1223 & 0.1210 & 2.4780 & 1.0651 & 1.0839 \\
0.0500 & 0.6106 & 0.6097 & 0.6097 & 0.6048 & 0.9513 & 0.7984 & 0.8054 \\
0.0650 & 0.7596 & 0.7585 & 0.7586 & 0.7545 & 0.6677 & 0.5300 & 0.5335 \\
0.0750 & -0.2022 & -0.2033 & -0.2033 & -0.2009 & 0.6691 & 1.1924 & 1.1903 \\
0.0850 & -0.9547 & -0.9580 & -0.9579 & -0.9566 & -0.2001 & 0.1391 & 0.1377 \\
0.0900 & -0.9655 & -0.9687 & -0.9687 & -0.9676 & -0.2173 & 0.1140 & 0.1122 \\
0.0950 & -0.6999 & -0.7017 & -0.7017 & -0.6974 & 0.3496 & 0.6071 & 0.6093 \\
0.1000 & -0.2404 & -0.2415 & -0.2415 & -0.2387 & 0.7006 & 1.1716 & 1.1717 \\
\hline
\end{tabular}

method has excellent accuracy and convergence characteristics. Compared with other numerical approaches that use step-by-step time integration, the present method has a unique advantage of being capable of producing results with very good and stable accuracy at a long time interval because the error accumulation is avoided due to the use of the analytical time series.

\section{References}

[1] S. Woinowsky-Krieger, "The effect of an axial force on the vibration of hinged bars," Journal of Applied Mechanics ASME, vol. 17, no. 1, pp. 35-36, 1950.

[2] R. Lewandowski, "Application of the Ritz method to the analysis of non-linear free vibrations of beams," Journal of Sound and Vibration, vol. 114, no. 1, pp. 91-101, 1987.

[3] M. Sathyamoorthy, Nonlinear Analysis of Structures, CRC Press, Boca Raton, Fla, USA, 1997.

[4] K. Y. Sze, S. H. Chen, and J. L. Huang, "The incremental harmonic balance method for nonlinear vibration of axially moving beams," Journal of Sound and Vibration, vol. 281, no. 3-5, pp. 611-626, 2005.

[5] M. M. Gadagi and H. Benaroya, "Dynamic response of an axially loaded tendon of a tension leg platform," Journal of Sound and Vibration, vol. 293, no. 1-2, pp. 38-58, 2006.

[6] R. A. Ibrahim and R. J. Somnay, "Nonlinear dynamic analysis of an elastic beam isolator sliding on frictional supports," Journal of Sound and Vibration, vol. 308, no. 3-5, pp. 735-757, 2007.

[7] E. Ozkaya and A. Tekin, "Non linear vibrations of stepped beam system under different boundary conditions," Structural Engineering and Mechanics, vol. 27, no. 3, pp. 333-345, 2007.

[8] S. H. Chen, J. L. Huang, and K. Y. Sze, "Multidimensional Lindstedt-Poincaré method for nonlinear vibration of axially moving beams," Journal of Sound and Vibration, vol. 306, no. 1-2, pp. 1-11, 2007.

[9] L. Azrar, R. Benamar, and R. G. White, "A semi-analytical approach to the non-linear dynamic response problem of S-S and C-C beams at large vibration amplitudes part I: general theory and application to the single mode approach to free and forced vibration analysis," Journal of Sound and Vibration, vol. 224, no. 2, pp. 183-207, 1999.

[10] L. Azrar, R. Benamar, and R. G. White, "A semi-analytical approach to the non-linear dynamic response problem of beams at large vibration amplitudes, part I: multimode approach to the steady state forced periodic response," Journal of Sound and Vibration, vol. 255, no. 1, pp. 1-41, 2002.

[11] A. Y. T. Leung and S. G. Mao, "Symplectic integration of an accurate beam finite element in non-linear vibration," Computers and Structures, vol. 54, no. 6, pp. 1135-1147, 1995.

[12] A. Y. T. Leung and S. G. Mao, "A symplectic Galerkin method for non-linear vibration of beams and plates," Journal of Sound and Vibration, vol. 183, no. 3, pp. 475-491, 1995. 
[13] A. Y. T. Leung and T. Ge, "Normal multi-modes of non-linear euler beams," Journal of Sound and Vibration, vol. 202, no. 2, pp. 145-160, 1997.

[14] R. Lewandowski, "Computational formulation for periodic vibration of geometrically nonlinear structures-part 1: theoretical background," International Journal of Solids and Structures, vol. 34, no. 15, pp. 1925-1947, 1997.

[15] R. Lewandowski, "Computational formulation for periodic vibration of geometrically nonlinear structures-part 2: numerical strategy and examples," International Journal of Solids and Structures, vol. 34, no. 15, pp. 1949-1964, 1997.

[16] M. I. Younis and A. H. Nayfeh, "A study of the nonlinear response of a resonant microbeam to an electric actuation," Nonlinear Dynamics, vol. 31, no. 1, pp. 91-117, 2003.

[17] S. A. Emam and A. H. Nayfeh, "On the nonlinear dynamics of a buckled beam subjected to a primaryresonance excitation," Nonlinear Dynamics, vol. 35, no. 1, pp. 1-17, 2004.

[18] S. A. Emam and A. H. Nayfeh, "Nonlinear responses of buckled beams to subharmonic-resonance excitations," Nonlinear Dynamics, vol. 35, no. 2, pp. 105-122, 2004.

[19] P. Ribeiro, "Hierarchical finite element analyses of geometrically non-linear vibration of beams and plane frames," Journal of Sound and Vibration, vol. 246, no. 2, pp. 225-244, 2001.

[20] R. Bellman, B. G. Kashef, and J. Casti, "Differential quadrature: a technique for the rapid solution of nonlinear partial differential equations," Journal of Computational Physics, vol. 10, pp. 40-52, 1972.

[21] CH. W. Bert, S. K. Jang, and A. G. Striz, "Nonlinear bending analysis of orthotropic rectangular plates by the method of differential quadrature," Computational Mechanics, vol. 5, no. 2-3, pp. 217-226, 1989.

[22] H. Zhong and Q. Guo, "Nonlinear vibration analysis of Timoshenko beams using the differential quadrature method," Nonlinear Dynamics, vol. 32, no. 3, pp. 223-234, 2003.

[23] J. Yang, S. Kitipornchai, and K. M. Liew, "Large amplitude vibration of thermo-electro-mechanically stressed FGM laminated plates," Computer Methods in Applied Mechanics and Engineering, vol. 192, no. 35-36, pp. 3861-3885, 2003.

[24] J. Yang, S. Kitipornchai, and K. M. Liew, "Non-linear analysis of the thermo-electro-mechanical behaviour of shear deformable FGM plates with piezoelectric actuators," International Journal for Numerical Methods in Engineering, vol. 59, no. 12, pp. 1605-1632, 2004.

[25] E. Manoach and P. Ribeiro, "Coupled, thermoelastic, large amplitude vibrations of Timoshenko beams," International Journal of Mechanical Sciences, vol. 46, no. 11, pp. 1589-1606, 2004.

[26] M.-H. Hsu, "Mechanical analysis of non-uniform beams resting on nonlinear elastic foundation by the differential quadrature method," Structural Engineering and Mechanics, vol. 22, no. 3, pp. 279-292, 2006.

[27] S. Tomasiello, "A DQ based approach to simulate the vibrations of buckled beams," Nonlinear Dynamics, vol. 50, no. 1-2, pp. 37-48, 2007.

[28] C. Mei, "Finite element displacement method for large amplitude free flexural vibrations of beams and plates," Computers and Structures, vol. 3, no. 1, pp. 163-174, 1973. 


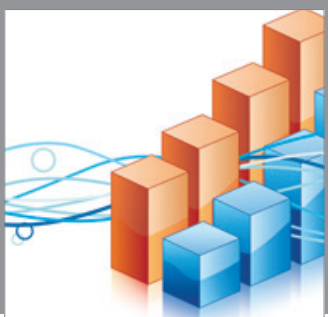

Advances in

Operations Research

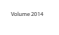

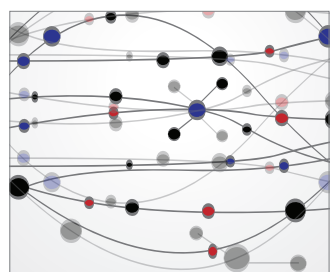

\section{The Scientific} World Journal
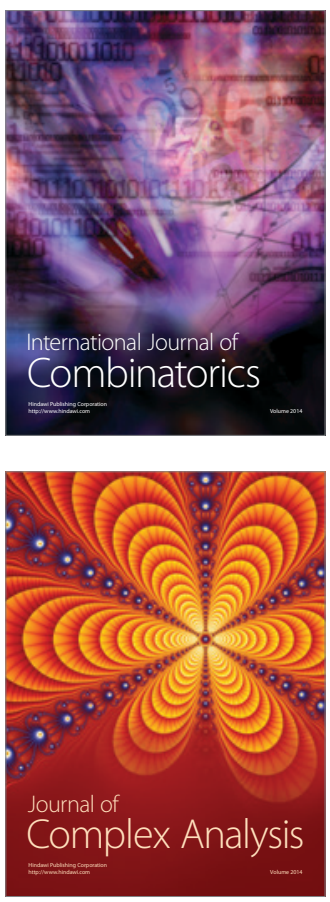

International Journal of

Mathematics and

Mathematical

Sciences
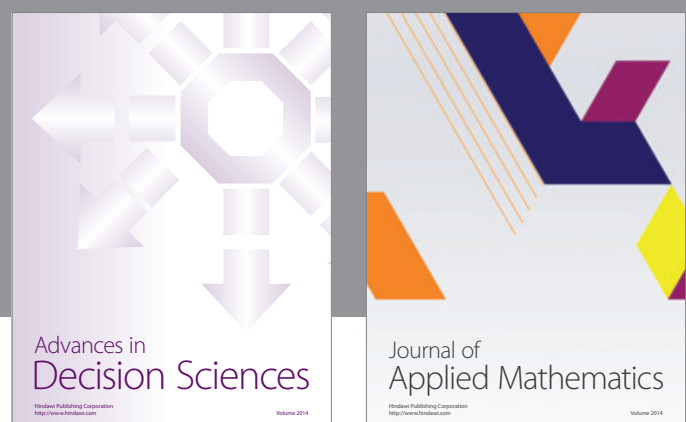

Journal of

Applied Mathematics
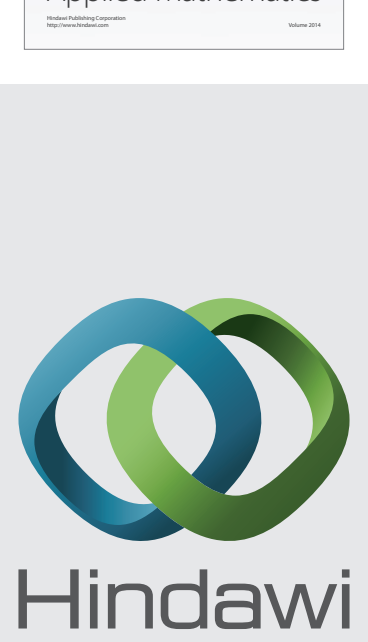

Submit your manuscripts at http://www.hindawi.com
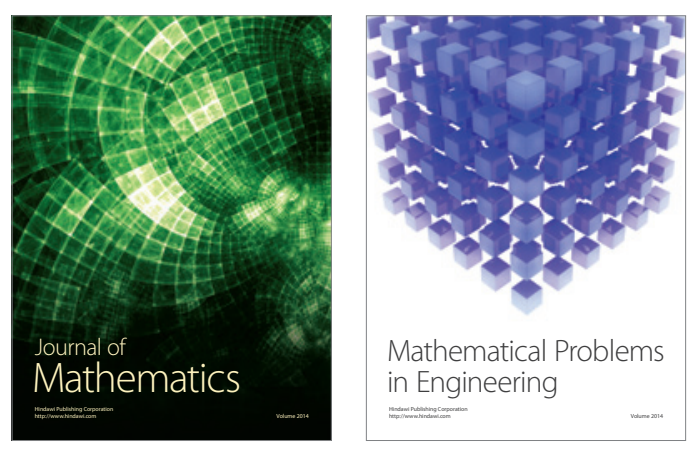

Mathematical Problems in Engineering
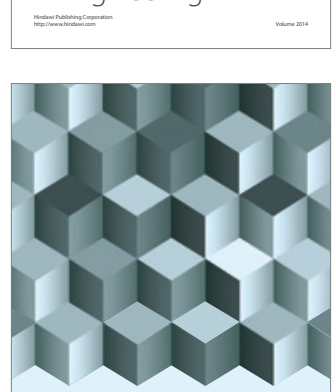

Journal of

Function Spaces
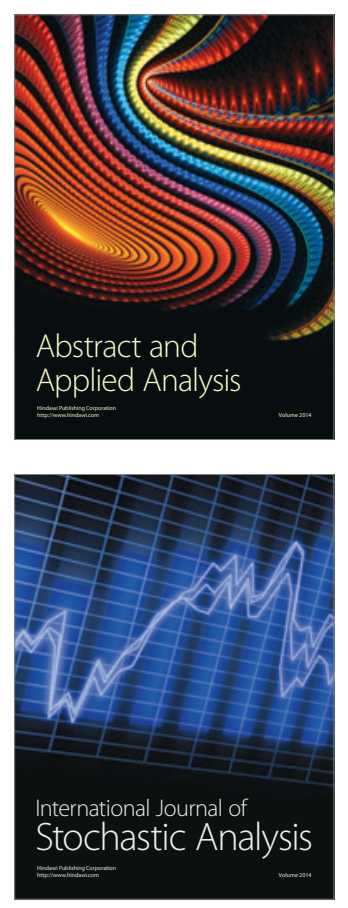

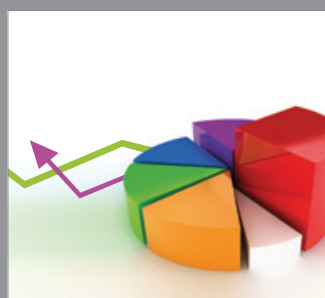

ournal of

Probability and Statistics

Promensencen
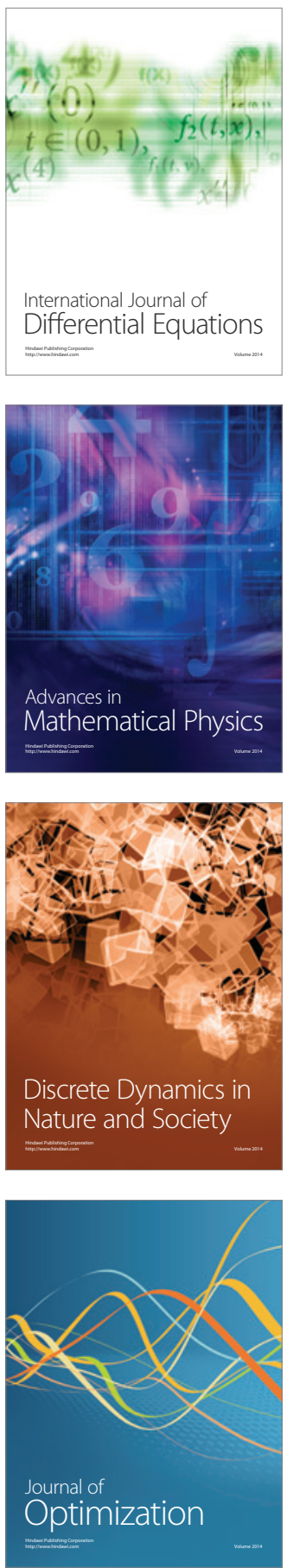